\title{
ETICA, ROBOTICA E LAVORO: Profili D'INFormatica GiURIDICA
}

1 Introduzione. 2 Robot e lavoro: Macchine intelligenti e autonomia decisionale. 3 Il lavoro dei robot: responsabilità e autonomia. 4 Tecnologie emergenti per una Sociatà 4.0. 5 I sistemi di decisione automatizzata e il trattamento dei dati personali. 6 Conclusioni. Referências.

\section{RESUMO}

Questo articolo trae spunto dalla Risoluzione del Parlamento europeo del 16 febbraio 2017 recante raccomandazioni alla Commissione concernenti norme di diritto civile sulla robotica L'avvento dell'Industria 4.0 ha incentivato ed esaltato l'uso di macchine intelligenti e dotate di gradi di autonomia sempre più crescenti. La ricerca sulla robotica, che oggi ha raggiunto livelli molto differenziati di applicazione, si appresta a diventare oggetto di interesse diffuso non solo nei processi produttivi, ma anche per i sistemi sociali funzionalmente differenziati. In particolare, il diritto si trova ad essere interessato dall'innovazione tecnologica più avanzata e dall'applicazione dell'Intelligenza Artificiale in quanto i robot, nelle scelte dell'Unione europea, sono destinatari di attribuzione di sempre maggiori responsabilità e di diritti. Questo solleva problemi, sia di carattere etico e sia di carattere giuridico, finora sconosciuti al diritto e sottovalutati dalla politica e dall'etica. Osservare dal punto di vista dell'Informatica giuridica questo fenomeno emergente consente di descrivere i modi attraverso i quali i sistemi sociali evolvono a seguito dell'irruzione delle nuove tecnologie nel sistema del diritto.

Palavras-chave: Roboetica. Robotica. Lavoro dei robot. Informatica giuridica. Diritti civili dei Robot.

\section{INTRODUZIONE}

La Risoluzione del Parlamento europeo del 16 febbraio 2017 recante raccomandazioni alla Commissione concernenti norme di diritto civile sulla robotica (2015/2103(INL))

* Dottore di ricerca in Scienze Giuridiche (Sociologia del diritto) nella Facoltà di Giurisprudenza dell'Università del Salento. Esperto di informatica giuridica, di metodologia della ricerca sociale e di sociologia del diritto. Attualmente si occupa dello studio dello Stato digitale, delle periferie della modernità e della loro organizzazione sociale svolgendo attività di ricerca su temi legati al diritto, alle teorie organizzative e alla ricerca sociale. Già professore a contratto nell'Università telematica TELMA-LA SAPIENZA di Roma, titolare degli insegnamenti di Sociologia della comunicazione elettronica e di Sistemi informativi nelle pubbliche amministrazioni. E-mail: <diviggianoluigi@gmail. com>. 
(EUROPEAN PARLIAMENT, 2017a), rubricata come Norme di diritto civile sulla robotica, ha posto l'accento su temi che, partendo dalla letteratura e dalla fantascienza, sono approdati all'interno di riflessioni che la società contemporanea comincia ad avvertire essere incombenti.

Lo studio sugli aspetti etici dei sistemi cyberfisici, la valutazione delle opzioni scientifiche e tecnologiche svolta a livello europeo e le relazioni di diverse Commissioni europee preliminari alla Risoluzione 2015/2103(INL), introducono alcune considerazioni poste a fondamento dei principi generali sulla robotica che si collocano nel sistema etico, giuridico, economico, dell'occupazione e degli affari sociali, dell'ambiente, della sanità, della sicurezza, dell'industria, dell'energia, della ricerca, del mercato e dei consumatori.

La riflessione su questi temi prende spunto da alcune considerazioni avanzate dalla Risoluzione del Parlamento europeo del 16 febbraio 2017, secondo le quali:

a) l'umanità si trova ora sulla soglia di un'era nella quale robot, bot, androidi e altre manifestazioni dell'intelligenza artificiale sembrano sul punto di avviare una nuova rivoluzione industriale, suscettibile di toccare tutti gli strati sociali, rendendo imprescindibile che la legislazione ne consideri le implicazioni e le conseguenze legali ed etiche, senza ostacolare l'innovazione;

b) negli ultimi duecento anni il tasso di occupazione è aumentato costantemente grazie agli sviluppi tecnologici. Lo sviluppo della robotica e dell'intelligenza artificiale è potenzialmente in grado di trasformare le abitudini di vita e lavorative, innalzare i livelli di efficienza, di risparmio e di sicurezza e migliorare il livello dei servizi, nel breve e medio termine. La robotica e l'intelligenza artificiale promettono di portare benefici in termini di efficienza e di risparmio economico non solo in ambito manifatturiero e commerciale, ma anche in settori quali i trasporti, l'assistenza medica, l'istruzione e l'agricoltura, consentendo di evitare di esporre esseri umani a condizioni pericolose;

c) l'invecchiamento è il risultato dell'allungamento della speranza di vita dovuto ai progressi nell'ambito del miglioramento delle condizioni di vita e della medicina moderna e che rappresenta una delle maggiori sfide politiche, sociali ed economiche del XXI secolo per le società europee;

d) i cambiamenti economici e le conseguenze per l'occupazione derivanti dalla robotica e dall'apprendimento automatico devono essere parimenti valutati. Nonostante i vantaggi innegabili apportati dalla robotica, essa può comportare una trasformazione del mercato del lavoro e rendere necessaria, di conseguenza, una riflessione sul futuro dell'istruzione, dell'occupazione e delle politiche sociali;

e) l'uso diffuso di robot potrebbe non portare automaticamente alla sostituzione di posti di lavoro, ma le mansioni meno qualificate nei settori ad alta intensità di manodopera potrebbero essere maggiormente esposte all'automazione;

f) la ricerca ha dimostrato che l'occupazione aumenta in modo particolarmente veloce nei settori caratterizzati da un maggiore impiego dei computer e che l'automazione dei posti di lavoro è potenzialmente in grado di liberare le persone dalla monotonia 
del lavoro manuale, consentendo loro di avvicinarsi a mansioni più creative e significative;

g) l'automazione richiede che i governi investano nell'istruzione e in altre riforme al fine di migliorare la ridistribuzione delle tipologie di competenze di cui avranno bisogno i lavoratori di domani;

h) lo sviluppo della robotica e dell'intelligenza artificiale eserciterà sicuramente un'influenza sul mondo del lavoro, il che potrebbe dare luogo a nuove preoccupazioni in materia di responsabilità ed eliminarne altre;

i) occorre chiarire la responsabilità giuridica per quanto concerne sia il modello di impresa sia le caratteristiche dei lavoratori, in caso di emergenza o qualora sorgessero problemi;

j) è possibile che a lungo termine l'intelligenza artificiale superi la capacità intellettuale umana.

Sulla base di questi assunti (considerata) e utilizzando le acquisizioni teoriche e di ricerca applicata sviluppate all'interno dell'Osservatorio IRIA (ITALY, 2017) e del Centro di Studi sul Rischio, ${ }^{1}$ il presente lavoro intende affrontare tematiche in merito alla necessità di norme che disciplinino il settore della robotica senza che tali regole compromettano il processo di ricerca, d'innovazione e di sviluppo nello stesso settore. Parallelamente si pone la questione della osservazione dell'evoluzione del diritto in funzione delle nuove acquisizioni tecnologiche che esercitano pressioni sulla società e sulla comunicazione, modificandole.

Questo impegno a riflettere diventa cogente soprattutto in vista della definizione di una Carta sulla robotica sociale a livello europeo che contenga, tra l'altro, questioni di responsabilità civile, di responsabilità giuridica derivante da azioni nocive dei robot che pongono problemi al diritto esigendo nuovi principi e regole. In particolare, l'utilizzo di robot nei settori produttivi pone problemi al sistema del lavoro e dell'occupazione, non solo in Europa, così come le tecnologie informatiche pongono problemi all'economia.

\section{ROBOT E LAVORO: MACCHINE INTELLIGENTI E AUTONOMIA DECI- SIONALE}

L'attuale letteratura tecnologica ed economica si occupa di descrivere con sempre maggiore frequenza come la ricerca sulle macchine intelligenti e la robotica abbia raggiunto livelli finora ipotizzati solo dalla fantascienza. All'inizio della quarta rivoluzione industriale (MAGONE; MAZALI, 2016) la robotica annoverava fra le applicazioni più evolute quelle realizzate in settori industriali che progressivamente hanno modificato il processo fordista di produzione sostituendo gli uomini con macchine sempre più capaci di assolvere a compiti e funzioni con una precisione, una velocità e una durata che il soggetto umano non può assicurare. Processi lavoravi pericolosi per l'uomo, venivano e vengono svolti da machine con possibilità di danno per i lavoratori molto remote. 
La robotica man mano trova impiego non solo in settori industriali "limitati" ma coinvolge progressivamente tutti i sistemi sociali, i sistemi produttivi e le singole persone. Le applicazioni robotiche oggi esercitano un forte impatto sulle attività umane: ludiche, relazionali, comunicative, lavorative, coinvolgendo le tecnologie, l'economia, l'etica, il diritto, la comunicazione. In questo modo, cercando di risolvere vecchi «problemi», ne introduce di nuovi. In particolare per il sistema del diritto.

Ormai la riflessione giuridica è sempre più concorde nel ritenere che servirebbero specifiche norme, anche internazionali, per disciplinare l'economia digitale sia per quanto riguarda l'aspetto giuridico che quello relativo ai diritti mentre è condiviso l'auspicio che almeno l'Europa riesca a predisporre un sistema di ordinamenti tale da garantire il rispetto dei diritti anche nell'era dell'economia digitale.

Con la Risoluzione del Parlamento europeo del 16 febbraio 2017 recante raccomandazioni alla Commissione concernenti norme di diritto civile sulla robotica (2015/2103(INL)), rubricata come Norme di diritto civile sulla robotica, l'Europa ha posto l'accento su temi che la società contemporanea comincia ad avvertire essere incombenti. Il discrimine che segna le semantiche, anche del diritto, tra l'esistente e ciò che si sta delineando nel campo dell'intelligenza artificiale applicata alle macchine è posto dal passaggio da Robot non autonomi eterodiretti (cose, automata) a Robot autonomi e intelligenti² dotati di personalità, elettronica, autopoietici.

Alcuni elementi abilitanti di queste nuove tecnologie concorrono a rendere possibili scenari in cui la robotica si evolve. La nuova generazione di algoritmi neurali, l'evoluzione dell'IoT da Internet of Things a Intelligent of Things, la produzione e la gestione di un quantitativo abnorme di dati digitali attraverso i Big Data, i Cloud, sono interessati e condizionati dalla produzione e gestione dell'energia necessaria al funzionamento, mentre tutto questo esige una diversa formazione delle generazioni presenti e future e impegna il diritto in nuove e ancora sconosciute forme di tutela e di regolazione di una materia che trova il suo fondamento nei linguaggi e nella comunicazione, trasformandoli.

La prospettiva tecnologica richiamata impegna anche l'etica e il lavoro in generale, ma in particolare coinvolge l'etica e il lavoro dei robot, eterodiretti o autonomi che siano. ${ }^{3} \mathrm{Nei}$ Considerata della Risoluzione del Parlamento europeo, si delinea l'idea che i Robot abbiano avviato una nuova rivoluzione industriale determinata dall'emergenza di diverse cause, ma che produce effetti la cui portata al momento può essere solo ipotizzata. I problemi riguardano il tasso di occupazione in rapporto all'affermarsi della robotica che incide sul mercato del lavoro, sull'istruzione e sulle politiche sociali a fronte di un costante invecchiamento della popolazione. La robotica pone questioni circa la responsabilità giuridica delle imprese, produttrici e utilizzatrici di tecnologia, ma anche dei lavoratori a cui sono richieste nuove competenze e capaci di svolgere nuove mansioni. Fino a toccare argomenti che mettono in discussione la dignità, l'autonomia e l'autodeterminazione umana. Allora si profila una domanda inquietante: cosa succede se dovesse sfuggire la capacità di verifica e di controllo 
delle macchine intelligenti e l'intelligenza artificiale dovesse superare quella umana?

Alcune affermazioni contenute nella Risoluzione del Parlamento europeo cercano di dare risposte ai tanti quesiti che la materia propone, ma le dichiarazioni di principio avanzate non riescono a fornire elementi stabili al diritto in grado di costruire un ordinamento sovranazionale rassicurante. Considerato che gli ordinamenti europei hanno interesse a mantenere il controllo delle norme regolamentari della robotica e dell'uso dell'intelligenza artificiale, anche al fine di non essere costretti ad adottare e subire norme stabilite da altri, affermare di ritenere fondamentali le Leggi di Asimov sulla robotica, ma che tali leggi non possano essere convertite in codice macchina procura una certa apprensione. Soprattutto se poi si afferma che "è necessaria una serie di norme che disciplinino in particolare la responsabilità, la trasparenza e l'assunzione di responsabilità e che riflettano i valori intrinsecamente europei, universali e umanistici che caratterizzano il contributo dell'Europa alla società; che tali regole non devono influenzare il processo di ricerca, innovazione e sviluppo nel settore della robotica."

$\mathrm{Al}$ momento solo la Corea del Sud con l'Intelligent robots development and distribution promotion Act del 2016, ${ }^{4}$ ha legiferato in materia, senza definire standard o principi universalmente validi, ma anche per questo l'Europa ritiene di poter

Svolgere un ruolo essenziale nella definizione dei principi etici fondamentali da rispettare per lo sviluppo, la programmazione e l'utilizzo di robot e dell'intelligenza artificiale e per l'inclusione di tali principi nelle normative e nei codici di condotta dell'Unione al fine di configurare la rivoluzione tecnologica in modo che essa serva l'umanità (EUROPEAN PARLIAMENT, 2017b, online).

Infatti l'Articolo 17 (Facilitazione della distribuzione di robot intelligenti a persone socialmente svantaggiate) afferma che il governo (coreano) deve predisporre le misure necessarie per facilitare lo sviluppo e la distribuzione di robot intelligenti per migliorare la comodità nell'uso di robot intelligenti, in modo che le persone socialmente svantaggiate, come i disabili, gli anziani e i lavoratori a basso reddito, possano godere di opportunità e benefici dall'usare liberamente tali robot. ${ }^{5}$

\section{IL LAVORO DEI ROBOT: RESPONSABILITÀ E AUTONOMIA}

Se consideriamo che l'autonomia di un robot può essere definita come la capacità di prendere decisioni e metterle in atto nel mondo esterno, indipendentemente da un controllo o un'influenza esterna (AA) ciò rende necessari nuovi principi e regole volte a chiarire la responsabilità legale dei vari attori per tutte quelle azioni e omissioni imputabili ai robot in quanto le decisioni autonome assunte da una macchina non consentirebbero di determinare qual è il soggetto a cui imputare la responsabilità del risarcimento né di esigere da tale soggetto la riparazione dei danni causati $(\mathrm{AB})$. 
Soprattutto in relazione alla istanza di creare una nuova categoria (AC) di persona giuridica, uno status giuridico specifico, anche se nel lungo termine, definita "persona elettronica" le norme tradizionali non sono sufficienti per attivare la responsabilità per i danni causati dalla nuova generazione di robot, perché questi possono essere dotati di capacità di adattamento e di apprendimento che implicano un certo grado di imprevedibilità nel loro comportamento e una capacità di apprendere in modo autonomo in base alle esperienze diversificate di ciascuno e di interagire con l'ambiente in modo unico e imprevedibile.

Proprio per individuare le migliori e più adeguate norme in grado di tutelare non solo da un punto di vista civilistico i soggetti giuridici e le persone, è fondamentale una più accurata classificazione delle macchine intelligenti al fine di declinare il confine che separa la macchina robotica eterodiretta da quella in grado di autodeterminarsi, qualità che decide aspetti giuridici, sociali, etici ed economici. Una possibile soluzione sarebbe quella di considerare i robot per i gradi di libertà di cui godono tecnicamente, per le funzioni che svolgono nelle applicazioni in cui sono impiegati ma anche in base ad una classificazione per livelli di autonomia che, partendo da un livello basico giunga a definire il livello più elevato in grado di descrivere la capacità decisionale autonoma e le variabili applicative a ciò collegate. Il gradino più alto della scala di autonomia proposta dal Gruppo di ricerca su IoT dell'Università Uniterma Sapienza di Roma, il livello 9 - descrive i Robot in grado di avere autonomia totale, formulare pensieri complessi, esercitare autocontrollo e possedere una capacità di apprendimento (Deep Learning) anche dai propri errori, non solo attraverso input esterni. ${ }^{6}$ Ogni livello di questa scala dell'autonomia comprende e assomma le caratteristiche del grado che lo precede e ne rappresenta un modello evolutivo.

La filosofia europea in materia di intelligenza artificiale considera che la tecnologia robotica dovrebbe integrare le capacità umane e non sostituirle, garantendo che gli uomini mantengano in qualsiasi momento il controllo sulle macchine intelligenti, proprio in applicazioni di quelle 3 leggi di Asimov sulla robotica a cui successivamente l'autore di "Io Robot" ne aggiungerà una quarta, superiore per importanza a tutte le altre ma valida solo per gli automi più sofisticati, definita legge zero: "Un robot non può recar danno all'umanità e non può permettere che, a causa di un suo mancato intervento, l'umanità riceva danno." Questa visione antropocentrica non sembra tenere conto del rischio insito nello sviluppo tecnologico delle macchine autonome dotate di intelligenza artificiale, ma forse intende solo esorcizzare una possibilità che ormai non si presenta più così remota. Gli sviluppi della tecnologia robotica autonoma e intelligente descrivono capacità umane sostituite dalle macchine (forse ancora non totalmente), paventano uno scarso, o nessun controllo sulle macchine intelligenti e autonome, scarsa capacità predittiva sulle attività cognitive dei robot e sul loro livello di autodeterminazione, fino alla riproduzione fisica. Si ipotizza, in questo modo, la nascita e lo sviluppo di un'etica non-umana. ${ }^{7}$

L'ormai famoso Rapporto del World Economic Forum, The Future of Jobs. Employment, Skills and Workforce Strategy for the Fourth Industrial Revolution del gennaio $2016^{8}$, tra l'altro, 
presenta una descrizione secondo la quale entro il 2020, nei 15 Paesi in cui si concentra il $65 \%$ della forza lavoro mondiale, le macchine porteranno via agli uomini oltre 5 milioni di posti di lavoro: stima che su 7.1 milioni di posti di lavoro perduti, solo 2 milioni saranno guadagnati. Questo alle condizioni dei parametri di stima del 2016.

Descrizioni come queste alimentano da tempo la paura che i robot possano portare via il lavoro all'uomo, creando schiere di disoccupati. Non è un timore nuovo in quanto questa preoccupazione è presente in ogni rivoluzione industriale. In tempi non recenti, proprio in relazione all'adozione di soluzioni lavorative affidate alla tecnologia e alle macchine ha alimentato riflessioni non banali. Keynes, nel suo saggio del 1930, Possibilità economiche per i nostri nipoti, colloca questa «disoccupazione tecnologica» nel 2030. Il fenomeno, per l'economista britannico, è causato dal fatto che «scopriamo sempre nuovi sistemi per risparmiare forza lavoro, $e$ li scopriamo troppo in fretta per riuscire a ricollocare quella forza lavoro altrove».

Nella prospettiva attuale, molti lavori tenderanno a diventare obsoleti, fino a sparire, mentre altri sorgeranno. Diverse posizioni che emergono da un sondaggio condotto nel 2014 negli Stati Uniti dal Centro di ricerche Pew, supportano questa idea (BATTIFOGLIA, 2016).

Tuttavia non c'è unanimità nel considerare le prospettive occupazionali del futuro rispetto all'affermarsi della robotica evoluta. Mentre da un lato si ipotizza che i lavori che i robot lasceranno fare agli uomini saranno quelli che richiedono idee e conoscenza (cioè solo coloro che tra gli umani avranno avuto una educazione d'eccellenza potranno competere con le macchine), dall'altro si profila la denuncia di una scarsa aderenza del sistema universitario alle mutate esigenze lavorative tanto da ritenere che sarà necessario adeguare il sistema educativo a tutti i livelli perché attualmente il sistema educativo non è ben posizionato per trasformarsi in modo da formare laureati in grado di competere con le macchine. Fino a giungere ad una visione più ottimistica secondo la quale i progressi dell'automazione cambieranno il lavoro ma non lo ridurranno (BATTIFOGLIA, 2016, p. 46-50).

Anche l'ONU manifesta una certa preoccupazione circa la possibilità che i sistemi robotizzati possano ridurre le possibilità di lavoro per gli umani (MARRO, 2016). Numerosi esempi di impiego di robot in attività lavorative lasciano pensare che il problema lavoro, che già oggi presenta elevate criticità, possa essere aggravato dall'uso di droni postini, di auto che si guidano da sole, da robot nel campo della sanità e dell'economia. Sul lato occupazione, l'utilizzo dei robot coinvolge soprattutto i Paesi emergenti: quelli che fino a ieri avevano sviluppato un'industria a basso valore aggiunto contando su una manodopera a costi di sopravvivenza. In un recente report, Robot and Industrialization in Developing Countries, ${ }^{9}$ dell'Unctad, la Conferenza delle Nazioni Unite sul commercio e lo sviluppo, mette in guardia Asia, Africa e America Latina circa l'impatto che l'era dei robot potrà avere sulla loro economia.

L'ONU segnala alcuni fattori importanti su cui bisogna puntare l'attenzione.

Il primo riguarda il cd reshoring. I Paesi sviluppati, che con la globalizzazione hanno delocalizzato produzioni a basso valore aggiunto nei Paesi in via di sviluppo, potrebbero decidere di riportarle in patria sostituendo i lavoratori low cost con robot tecnologicamen- 
te avanzati. È un rischio non remoto non solo per i lavoratori ma anche per le imprese in quanto il rimpatrio delle produzioni delocalizzate costringerà le aziende che vorranno restare competitive a puntare sull'automazione avanzata.

L'ONU consiglia, poi, ai Paesi emergenti di abbracciate la rivoluzione digitale a partire dai banchi di scuola. «Bisogna ridisegnare i sistemi educativi - spiega il report - in modo da creare le competenze manageriali e professionali necessarie a lavorare con le nuove tecnologie».

Un ulteriore indicazione suggerisce: se non potete battere i robot, puntate su di loro. Un mix innovativo di lavoro uomo-macchina permetterebbe ai Paesi emergenti di mantenere la competitività della propria struttura industriale, magari evitando il temuto reshoring. «In risposta all'invecchiamento della popolazione e all'aumento del costo del lavoro, che sta erodendo i vantaggi della manodopera manuale low cost, fin dal 2013 la Cina ha acquistato più robot industriali di ogni altro Paese», spiega lo studio dell'Unctad.

Partendo da questa visione complessa e variegata si sta facendo strada anche l'idea secondo la quale la robotica consentirà di ridurre le ore lavorative così che la settimana lavorativa diventerà più breve, ${ }^{10}$ saranno eliminati lavori monotoni, pericolosi, ripetitivi e sgradevoli. Lo stesso livello retributivo, invece di diminuire potrebbe consentire un livello più elevato di remunerazione del lavoro, certamente più qualificato. Necessariamente questo avrà un impatto sul diritto del lavoro e sulle relazioni sindacali che dovranno orientarsi affinché siano adeguate le norme, aggiornate la negoziazione, previsti contratti nuovi e relazioni industriali basate su un concetto di lavoro che è destinato a cambiare in maniera significativa.

Questa descrizione consente di avanzare alcune considerazioni sul lavoro dei robot. In primo luogo l'avvento dei robot sempre più intelligenti e preformati può causare una progressiva perdita di posti di lavoro. Parallelamente, i lavori necessari per produrre tecnologie di livello elevato, come anche costruire i robot, richiederà un incremento di nuove professionalità e, di conseguenza, un aumento di posti di lavoro. Ma il livello più auspicabile è quello che vede l'uomo lavorare con il robot per la costruzione di un nuovo umanesimo che potremmo indicare come umanesimo tecnologico o Cyberumanesimo. Il diritto, in ogni caso e a tutti i livelli, sarà chiamato in causa e sarà orientato a funzionare secondo i criteri dell'autopoiesi dei sistemi sociali: per affrontare nuovi problemi sarà costretto a evolvere. Per descrivere questo processo possiamo prendere in considerazioni due prospettive: l'evoluzione endogena e quella esogena. Nella prospettiva dell'evoluzione endogena, comprensibile mediante la teoria dei sistemi sociali, l'evoluzione di un sistema dipende dalla complessità dell'ambiente, ma anche dalla propria complessità, cioè dalla propria differenziazione interna. La soddisfazione di tre diverse funzioni all'interno del sistema del diritto rende possibile questo tipo di evoluzione e cioè:

1. produzione di possibilità di tipo nuovo all'interno del sistema, per il resto invariato (VARIAZIONE). In questo caso l'irruzione della tecnologia nella società pone 
istanze giuridiche che il sistema del diritto assume come nuove, come variazioni;

2. selezione delle possibilità utilizzabili, ed esclusione di quelle inutilizzabili (SELEZIO$\mathrm{NE}$ ). In questa fase il diritto seleziona ciò che la robotica, l'intelligenza artificiale e le nuove tecnologie renderanno utilizzabile dal diritto, proponendo nuove forma di normazione, introducendo nuovi diritti e nuovi modi di soddisfarli o di renderli esigibili;

3. stabilizzazione delle possibilità utilizzabili nella struttura del sistema (STABILIZZAZIO$\mathrm{NE}$ ). Ciò che il sistema del diritto assume attraverso la selezione e resiste al vaglio della prova giuridica, si stabilizza fino a che il nuovo non irromperà sulla scena, ri-innescando il processo evolutivo del diritto (LUHMANN, 1990, p. 36-40).

Affinché la variazione, la selezione e la stabilizzazione vengano attualizzate internamente al sistema è necessario essere in presenza di un sistema che sia interessato, relativamente ad alcune sue parti isolabili, alle spinte, alle informazioni e alla "causalità direttamente determinante", provenienti dall'ambiente per acquisire, quindi, la capacità di produrre grandi effetti relativamente a piccole cause, innescando un procedimento di rafforzamento interno dell'effetto. ${ }^{11}$

\section{TECNOLOGIE EMERGENTI PER UNA SOCIETÀ 4.0}

La caratteristica peculiare della robotica mossa da algoritmi neurali e dotata di IA risiede nella capacità delle macchine di effettuare un apprendimento automatico. L'apprendimento automatico da parte di strutture tecnologiche valutabili come ricadenti dell'ambito della robotica, è onnipresente. Dalla diagnosi medica, al riconoscimento vocale e dal riconoscimento della calligrafia alle raccomandazioni automatizzate di trading, le tecniche di machine learning vengono utilizzate per prendere decisioni critiche sul business e sulla vita in ogni momento della giornata. Ogni problema di apprendimento automatico è unico, quindi può essere difficile gestire i dati grezzi, identificare le caratteristiche chiave che influiscono sul modello, formare più modelli ed eseguire valutazioni del modello stesso. Ai fini del presente lavoro diventa fondamentale richiamare l'attenzione sulle caratteristiche dell'apprendimento automatico delle macchine (Machine Learning) che può essere supervisionato ${ }^{12}$ e non supervisionato, ${ }^{13}$ che può essere rinforzato ${ }^{14}$ fino a giungere all'utilizzo di reti neurali artificiali ${ }^{15}$ in grado di dotare la macchina di capacità di apprendimento tali da rendere autonome le proprie decisioni.

Nel 2017, Dell Technologies ha collaborato con il gruppo di ricerca indipendente sui futures, l'Institute for the Future (IFTF), per esplorare le tecnologie emergenti che modelleranno il futuro dell'esperienza umana nel prossimo decennio e gli impatti e le implicazioni specifiche che avranno su società e lavoro. Per eseguire ciò, IFTF ha fatto affidamento su propri studi pluridecennali sul futuro del lavoro e della tecnologia, ha realizzato interviste approfondite con le principali parti interessate $\mathrm{e}^{16}$ ha tenuto in considerazione le opinioni e le 
idee generate da focus svolti con gruppi diversificati di esperti provenienti da tutto il mondo.

Al principio, pochissime persone hanno avuto la capacità di capire in anticipo il ritmo con cui Internet si sarebbe diffuso in tutto il mondo o di ipotizzare l'impatto che la Rete avrebbe avuto nel rimodellare affari e cultura. Eppure, come ha scritto il giornalista Oliver Burkeman nel 2009, "senza che la maggior parte di noi se ne accorga quando è successo, il web è passato dall'essere una strana nuova curiosità ad una condizione di fondo della vita di tutti i giorni" (BURKEMAN, 2009, online).

Le tecnologie emergenti di oggi, agli occhi della gente comune, appaiono anche come strane, nuove curiosità. Intelligenza artificiale $(\mathrm{AI}),{ }^{17}$ realtà aumentata e virtuale, robot domestici e cloud computing, solo per indicare alcune delle sofisticate tecnologie in fase di avanzato sviluppo, stanno catturandogli gli interessi e l'immaginazione di molti. Le capacità avanzate delle odierne tecnologie emergenti spingono molti ricercatori, imprenditori e imprese a immaginare orizzonti del futuro in cui l'impatto sulla società dei loro effetti sarà a dir poco trasformativo. In un recente workshop di esperti condotto da Dell Technologies e dall'Institute for the Future (IFTF), i partecipanti hanno suggerito che le tecnologie in gioco nel prossimo decennio potrebbero "risolvere alcuni dei problemi irrisolvibili che l'umanità ha affrontato per così tanto tempo", offrire l'opportunità di "aumentare la produttività [in modo tale] che tutti i nostri bisogni di base [siano curati]" e "fondamentalmente riformulare" i riferimenti culturali di cosa significhi essere una persona.

Tutto questo indipendentemente dal fatto che non è scontato che le citate tecnologie emergenti realizzeranno queste ambiziose possibilità. L'orizzonte temporale di questa previsione è il 2030. Nello sviluppo sociale considerato, le tecnologie plausibilmente si intersecheranno e interagiranno con potenti forze demografiche, economiche e culturali così da sconvolgere le condizioni della vita quotidiana oggi conosciute e tanto da ridefinire il numero di persone che vivranno e lavoreranno nel 2030. Di conseguenza, molte organizzazioni e persone navigheranno verso acque inesplorate nel prossimo decennio, incerto su ciò che ci aspetta. Nello studio Dell: Digital Transformation Index, realizzato in collaborazione con 4.000 dirigenti, responsabili ai massimi livelli distribuiti in tutto il mondo, il $45 \%$ di loro afferma di essere preoccupato di diventare obsoleto in soli 3-5 anni; quasi la metà di essi non sa come sarà il settore di propria competenza in soli tre anni di tempo e il 73\% di loro crede di aver bisogno di essere più 'digitale' per avere successo in futuro.

Il Rapporto previsionale Dell conclude che, nel prossimo decennio, le tecnologie emergenti sosterranno la formazione di nuove partnership uomo-macchina che sfruttano al massimo i rispettivi punti di forza complementari. Queste partnership miglioreranno le attività quotidiane attorno al coordinamento delle risorse e all'apprendimento immediato, che resetterà le aspettative sul lavoro e imporrà alle strutture aziendali di adattarsi alle capacità in espansione dei team uomo-macchina. ${ }^{18}$

Tutto questo mentre oggi ancora si discute sul tema Diritti umani nell'era della cittadinanza digitale utilizzando strumenti inadeguati e che denunciano l'usura del tempo. 
Il passaggio da una società 2.0 verso un assetto sociale che ormai si configura come $4.0^{19}$ (che già compie incursioni verso un modello 5.0), con l'avvento della robotica e delle applicazioni più evolute dell'intelligenza artificiale, si può osservare anche attraverso la descrizione di come il diritto si occupa di problemi che con grande fatica sono stati riportati nel sistema giuridico, ma le cui soluzioni giuridiche già presentano segni di obsolescenza evidenti. ${ }^{20}$

\section{I SISTEMI DI DECISIONE AUTOMATIZZATA E IL TRATTAMENTO DEI DATI PERSONALI}

In merito al trattamento dei dati personali, l'Europa ha approvato il Regolamento Europeo 679/2016 (EUROPA, 2016) ${ }^{21}$ rubricato come: Regolamento (UE) 2016/679 del Parlamento europeo e del Consiglio, del 27 aprile 2016, relativo alla protezione delle persone fisiche con riguardo al trattamento dei dati personali, nonché alla libera circolazione di tali dati e che abroga la direttiva 95/46/ CE (regolamento generale sulla protezione dei dati).

Relativamente alla robotica e all'intelligenza artificiale è necessario considerare le possibili implicazioni che intercorrono tra il trattamento dei dati personali e i sistemi di decisione automatizzata. La General Data Protection Regulation (GDPR) rappresenta l'orizzonte giuridico e operativo con cui privati e istituzioni pubbliche devono confrontarsi. La domanda, allora, si pone in questi termini: quali sono le implicazioni giuridiche dell'impatto della GDPR con i sistemi robotici? Se si considera che non si tratta soltanto di possibilità future in quanto, attualmente, numerose aziende stanno investendo nei sistemi di Machine Learning e di intelligenza artificiale, il problema si pone già oggi ed è urgente. Mentre la previsione della GDPR si applica a qualsiasi sistema in grado di assumere una decisione automatizzata, il principio fondamentale è che una funzione unicamente automatizzata in grado di comportare delle decisioni, degli effetti giuridici sull'individuo, è vietata. ${ }^{22}$ In questa prospettiva giuridica dobbiamo tener conto di alcuni elementi nella valutazione di queste tecnologie.

Per primo, questo divieto non trova applicazione in riferimento a qualsiasi tecnologia in grado di produrre una decisione automatizzata: deve essere una decisione unicamente automatizzata e deve essere una decisione in grado di produrre effetti giuridici rilevanti sull'individuo come, per esempio, può essere la stipula o meno di un contratto.

In secondo luogo, ci sono delle eccezioni a questo divieto. L'eccezione più rilevante è data dal trattamento che è necessario all'esecuzione del contratto tramite il sistema automatizzato. Ebbene questo termine è interpretato in modo restrittivo dei garanti europei. Bisogna considerare ciò che è strettamente necessario all'esecuzione del contratto e la tipologia di profilazione che il sistema di intelligenza artificiale può compiere in quanto questa attività di trattamento dei dati personali potrebbe andare oltre ciò che è strettamente necessario e contravvenire il principio di necessità e non eccedenza. A meno che non esista la possibilità 
di dimostrare che questa maggiore profilazione, quindi la violazione di un principio ribadito dalla GDPR, sia in grado di dare risultati più efficaci, più veloci e maggiormente corretti, anche nell'interesse del singolo individuo interessato. Un problema di questa eccezione, è che non trova applicazione con riferimento ai dati sulla salute e, quindi, potrebbe avere un impatto negativo sulle compagnie assicurative che dovranno fare affidamento sulla seconda eccezione, cioè sul consenso esplicito dell'interessato. Questa è una eccezione abbastanza delicata perché si fa dipendere l'utilizzo della tecnologia da una decisione discrezionale dell'individuo che potrebbe anche sottrarsi a questa decisione.

C'è da considerare poi che bisogna avere trasparenza nella logica del funzionamento della tecnologia rispetto alle informazioni che sono processate per raggiungere la decisione sia rispetto alla fonte di queste informazioni e sia rispetto all'impatto della decisione sul singolo individuo. Questo è abbastanza rilevante perché non si può ottenere un consenso, non si può ottenere una informativa sulla privacy che faccia riferimento a qualsiasi tipologia di tecnologia che comporta una decisione automatizzata perché la descrizione dell'informativa deve essere basata sulla tecnologia che può essere utilizzata per questo processo.

Esiste, e può essere esercitato, un diritto di opposizione da parte dei singoli individui al trattamento dei dati. Questo comporta la necessità di disporre di un procedimento che consenta una verifica manuale della decisione. Ma questo comporta anche la possibilità/ necessità di ricostruire il ragionamento che la macchina ha fatto per raggiungere una determinata conclusione/decisione. Questa problematica non è di poco conto in presenza di sistemi di intelligenza artificiale che diventeranno sempre più complessi e autonomi.

Un ultimo elemento richiama la necessità di realizzare un determinato Data Protection Impact Assessment ${ }^{23}$ sulla tecnologia utilizzata come previsto dall'Art. 35 della GDPR che introduce il concetto di Data Protection Impact Assessment (DPIA). Ciò significa che bisogna valutare l'invasività della tecnologia utilizzata, assicurarsi che vi siano tutele sufficienti per gli individui, verificare se la tecnologia sia stata adeguatamente testata e imputare le responsabilità eventuali. Questo tipo di verifiche costituisce un elemento su cui insistono molto i Garanti europei poiché queste tecnologie sono il presente e saranno sempre più il futuro ma bisogna essere in grado di utilizzarle correttamente anche sotto il profilo del trattamento dei dati personali.

La stessa proposta di Carta sulla robotica contenuta nella Risoluzione del Parlamento Europeo del febbraio 2017, in particolare la parte destinata ai progettisti, si muove in questa direzione quando stabilisce che "Il progettista dourebbe introdurre funzionalità di "privacy by design" (tutela della vita privata fin dalla progettazione), in modo da garantire la sicurezza delle informazioni private e assicurare che queste ultime siano utilizzate soltanto in modo appropriato". Questo significa che la progettazione dei robot deve recepire i principi di protezione dei dati personali in modo da garantire la sicurezza degli stessi e assicurare che essi siano utilizzati soltanto in modo appropriato. In particolare prevedendo l'adozione di misure tecniche tali che il trattamento dei dati ad opera di un robot sia conforme alle prescrizioni del Regolamento 679/2016 (art. 
25). La previsione di una sempre maggiore autonomia dei robot e la capacità di recepire e produrre dati da parte delle macchine complica il quadro di riferimento sul lato del diritto e pone interrogativi circa la reale capacità delle norme di tutelare i dati personali in riferimento alla robotica avanzata.

\section{CONCLUSIONI}

Ciascuno dei temi trattati potranno contribuire alla costruzione di una "Carta sociale della robotica" (se possibile). La "Carta" potrà essere lo strumento per tenere alta l'attenzione di tutti sul tema della robotica e dei diritti ad essa collegati e costituire il filo conduttore di successivi approfondimenti. Il Parlamento europeo sottolinea come la prossima legislazione in materia di robotica dovrebbe tener conto dei principi sanciti nella Carta sulla robotica la quale svolge una funzione complementare alla robot law, quest'ultima diretta a regolamentare la coesistenza tra uomo e macchine. Cioè l'avvento dei robot cognitivi, indipendenti e, in qualche misura, imprevedibili, apre alla riflessione sull'opportunità della creazione di una nuova branca del diritto (STANZIONE, 2010).

Un diritto che attraverso l'uso dei robot può ottenere un vantaggio di gran lunga maggiore di quello che in, tempi anche recenti, si riteneva potessero procurare i cd sistemi esperti. ${ }^{24}$ L'idea secondo la quale un sistema informativo costruito su una "base di conoscenza" consistente in un insieme di informazioni specialistiche di un dato dominio (il diritto per esempio) potesse svolgere funzioni che richiedono specifica competenza (come un giudice) fino a giungere alla produzione di una sentenza, ad oggi non trova applicazione fattuale. Salvo l'automazione di alcuni procedimenti che operano su un dataset specifico, con specifiche e limitate funzionalità, in "collaborazione" con gli operatori umani (SARTOR, [20--], p. 298-304)., in modo puramente deduttivo.

Una recente notizia del settembre 2017 che arriva dalla provincia di Jiangsu, Cina, racconta di una truppa di avvocati-robot impiegati nella valutazione di casi giudiziari, soprattutto violazioni del codice della strada, che hanno comminato 541 condanne in riferimento a 15.000 casi trattati (ROMANDINI, 2017).

Il 30 ottobre 2017 Wired (MOSCA, 2017) pubblica un articolo con cui si afferma che l'Arabia Saudita ha concesso la cittadinanza all'androide Sophia, subito diventato una notizia sensazionale di internet. Da quando Hanson Robotics, con sede a Hong Kong, l'ha attivata per la prima volta nel 2015, l'androide ha percorso le tappe di "The Tonight Show with Jimmy Fallon” nell'aprile del 2017, del Web Summit a Lisbona e del Wired Next Fest di Firenze. A ottobre, è diventata la prima macchina a ricevere la cittadinanza paradossalmente in una nazione, l'Arabia Saudita, dove le donne non hanno ancora il riconoscimento di diversi diritti fondamentali.

Mentre a febbraio 2017 il Parlamento europeo proponeva il riconoscimento di personalità elettronica per i robot intelligenti e autonomi, già a ottobre dello stesso anno, anche 
se simbolicamente e per fini esclusivi di promozione commerciale, veniva conferita la cittadinanza a Sophia, un robot umanoide.

La riflessione su questi temi pone, allora, la necessità di considerare come prioritari alcuni elementi:

1. L'Europa è preoccupata di individuare standard in materia tipicamente europei per contrastare la concorrenza esterna in materia di robotica e di AI. Il problema, però è la armonizzazione degli standard lavorando per un sistema quanto più condiviso e comune.

2. Resta impregiudicata la necessità di individuare gli elementi comuni per una governance "sicura" del sistema della robotica, dell'AI e dell'innovazione tecnologica.

3. La preoccupazione di una preminenza imminente delle macchine e dell'AI sull'uomo deve essere stemperata attraverso norme giuridiche, tecniche ed etiche in grado di garantire, fin dalla progettazione, macchine "sicure" per quanto questo possa avere senso.

4. Occorre pensare un nuovo welfare in cui le risorse economiche possano essere fornite dal complesso sistema delle aziende e delle macchine impegnate in attività lavorative.

5. Occorre pensare un adeguato diritto del lavoro e diverse relazioni industriali sia per la tutela dei lavoratori e sia per la tutela di coloro che il lavoro non riescono a trovarla.

6. Occorre intervenire nel sistema dell'istruzione a tutti i livelli in modo da formare le generazioni presenti e quelle future per far acquisire conoscenze, skill e saperi che possano consentire di non essere marginalizzati nel mutato scenario lavorativo complessivamente inteso.

7. Sviluppare le politiche e le norme necessarie a promuovere la conoscenza tecnologica ai più alti livelli senza trascurare le conoscenze umanistiche che rappresentato il patrimonio accumulata dell'umanità fina ad oggi

8. Non escludere la Pubblica amministrazione dai processi di automazione soprattutto in quei settori che erogano servizi ai cittadini.

Anche sul versante del diritto il percorso sembra segnato ma le difficoltà sono notevoli e non sempre immaginabili avendo a disposizione le acquisizioni evolutive ad oggi raggiunte.

\title{
ETHICS, ROBOTICS AND LABOR: A COMPUTER LAW PROFILE
}

\begin{abstract}
This article brings to discussion the European Parliament Resolution of 16 February 2017 advancing a recommendation to the Commission concerning the norms of Civil Law on
\end{abstract}


robotics. The advent of Industry 4.0 has further incentivized and highlighted the use of intelligent and autonomous machines. The research on robotics, which currently reaches very different levels of application, is about to become the object of different interests, not just in productive processes, but also for functionally differentiated social systems. In particular, Law is interested in more advanced technological innovation and in the application of artificial intelligence, since robots, as an option of the European Union, are the addressees of attributions growing in responsibility and of rights. This raises problems, be them of an ethical or of a legal character, so far unknown to Law and underestimated by Politics and Ethics. To observe this emerging phenomenon from the point of view of Computer Law allows for the ways through which the social systems evolve to be described following the intrusion of new technologies in the system of the Law.

Keywords: "Roboethics". Robotics. Labor of robots. Computer Law. Civil rights of the robots.

\section{ÉTICA, ROBÓTICA Y TRABAJO: UN PERFIL DE INFORMÁTICA JURÍDICA}

\section{RESUMEN}

Este artículo trae a discusión la Resolución del Parlamento Europeo de 16 de febrero de 2017 avanzando una recomendación a la Comisión a respecto de las normas de Derecho Civil sobre robótica. El adviento de la Industria 4.0 a incentivado aún más y resaltado el uso de máquinas autónomas e inteligentes. La investigación a respecto de la robótica, que a presente alcanza diferentes niveles de aplicación, está prestes a tornarse el objeto de diferentes intereses, no solamente en procesos productivos, pero también para sistemas sociales funcionalmente diferenciados. En particular, el Derecho está interesado en innovaciones tecnológicas más avanzadas y en la aplicación de la inteligencia artificial, ya que robots, como una opción de la Unión Europea, son los destinatarios de atribuciones que crecen en responsabilidad y de derechos. Eso plantea un problema, sea de carácter ético o de carácter legal, hasta ahora desconocido del Derecho y subestimado por la Política y la Ética. Observar este fenómeno emergente desde el punto de vista de la Informática Jurídica permite describir los medios a través los cuales los sistemas sociales evolucionan después de la intrusión de nuevas tecnologías en el sistema del Derecho.

Palabras-clave: "Roboética". Robótica. Trabajo de robots. Informática Jurídica. Derechos civiles de los robots.

1 CSR, Università del Salento, Dipartimento di Scienze Giuridiche, Direttore: Prof. Raffaele De Giorgi.

2 Il superamento del dualismo creatosi tra IA forte-IA debole porta a ritenere che la visione di una intelligenza light non esclude un'autonomia delle macchine che, in questa prospettiva, riposa su processi di elaborazione 
di dati e apprendimento; nell'interagire con l'ambiente, le macchine apprendono e si emancipano, assumendo decisioni e comportamenti che non sono predeterminati da un software. L'autonomia di un robot denota pertanto la capacità di prendere decisioni e metterle in atto nell'ambiente, indipendentemente da un controllo esterno; tale autonomia è di natura puramente tecnologica. L'autonomia dei robot esclude - o, quanto meno, riduce - la possibilità di un loro utilizzo strumentale da parte dell'uomo, e solleva la questione dell'applicabilità delle categorie giuridiche esistenti e dell'eventuale necessità di creare una nuova categoria con caratteristiche specifiche e implicazioni proprie. Cf. (MARINI; APREA, 2015; SEARLE, 1980).

3 Nell'ambito degli studi di intelligenza artificiale è emersa l'esigenza di elaborare algoritmi che possano allineare le scelte dei robot ai valori condivisi dagli uomini, pur mantenendo la capacità di adattare continuamente il loro comportamento a nuove situazioni. Sotto questo aspetto, la progettazione dovrebbe contemplare un algoritmo di apprendimento che possa far distinguere alla macchina i comportamenti 'giusti' da quelli 'sbagliati'. Un siffatto modello ha una radice nel paradigma del software utilizzato come 'codice' (norma) del comportamento, secondo il quale le istruzioni che lo compongono dovrebbero conformare il comportamento (umano o delle macchine) alle norme giuridiche. Cf. (SANTOSUOSSO; BOSCARATO; CAROLEO, 2012).

4 Statute of the Republic of Korea, Intelligent Robots Development and Distribution Promotion Act, 2016. "Article 1 (Purpose) - The purpose of this Act is to contribute to enhancing the quality of life of citizens and the national economy by establishing and promoting a policy on the sustainable development of the intelligent robot industry to facilitate the development and distribution of intelligent robots and lay down the foundation therefor." Available in: <http://elaw.klri.re.kr/eng_service/lawTwoView.do?hseq=39153>.

5 Statute of the Republic of Korea, Intelligent Robots Development and Distribution Promotion Act, 2016 Article 17 (Facilitation of Distribution of Intelligent Robots to Socially Disadvantaged People) The Government shall prepare measures necessary for facilitating the development and distribution of intelligent robots to improve convenience in using intelligent robots so that socially disadvantaged people, such as the disabled, the elderly, and low-income earners, can enjoy opportunities for and benefits from using freely such robots. Available in: <http://elaw.klri.re.kr/eng_service/ lawTwoView.do?hseq=39153>. Questo articolo si avvicina ai punti 31 e 32 dei Principi generali riguardanti lo sviluppo della robotica e dell'intelligenza artificiale per uso civile, della citata Risoluzione del Parlamento europeo del 16 febbraio 2017, il quale afferma che: "la ricerca e lo sviluppo di robot per l'assistenza agli anziani sono diventati, nel tempo, più diffusi ed economici, permettendo così di produrre dispositivi dotati di maggiori funzionalità e più facilmente accettati dai consumatori; evidenzia l'ampia gamma di applicazioni di tali tecnologie utilizzate per la prevenzione, l'assistenza, il monitoraggio, lo stimolo e l'accompagnamento degli anziani, come pure delle persone affette da demenza, disturbi cognitivi o perdita della memoria" (31) mentre ritiene "che la sostituzione del fattore umano con i robot potrebbe, da una parte, disumanizzare le pratiche di accudimento, ma riconosce, d'altra parte, che i robot potrebbero svolgere compiti di assistenza automatizzati e agevolare il lavoro degli assistenti sanitari, migliorando, nel contempo, le cure fornite dal personale sanitario (32).

6 Nella prospettiva machine learning i comportamenti non sono impartiti dalle istruzioni di un software, ma sono appresi dall'elaborazione dell'esperienza in funzione di un criterio di miglioramento delle prestazioni; tali comportamenti, dunque, pur essendo riconducibili a un modello di apprendimento, non sono prevedibili nel loro specifico dispiegarsi. Un robot può assumere un comportamento iniziale definito da un software, ma ha anche la capacità di modificarlo dopo aver elaborato un insieme di dati rilevati, attraverso i suoi sensori, nell'ambiente di riferimento. In simili condizioni, appare alquanto complesso per un soggetto conoscere quali dati verranno raccolti e quale sarà il nuovo comportamento della macchina. Cf. (MORO, 2015).

7 La Roboetica rappresenta quella branca dell'etica dei robot che dovrà indagare i limiti di accettabilità etica delle pratiche riguardanti i robot ed avrà il compito di creare un consenso di base. Cf. (STANZIONE, 2010). Nell'ambito degli studi di intelligenza artificiale è emersa l'esigenza di elaborare algoritmi che possano allineare le scelte dei robot ai valori condivisi dagli uomini, pur mantenendo la capacità di adattare continuamente il loro comportamento a nuove situazioni. In relazione a ciò è diffusa l'idea secondo la quale la progettazione dovrebbe contemplare un algoritmo di apprendimento che possa far distinguere alla macchina i comportamenti 'giusti' da quelli 'sbagliati'. Un siffatto modello ha una radice nel paradigma del software utilizzato come 'codice' (norma) del comportamento, secondo il quale le istruzioni che lo compongono dovrebbero conformare il comportamento (umano o delle macchine) alle norme giuridiche. Cf. (SANTOSUOSSO; BOSCARATO; CAROLEO, 2012).

8 Cf. (WORLD ECONOMIC FORUM, 2016). 
9 Cf. il Report: (ROBOTS..., 2016).

10 L'idea, in Italia, non è nuova. In un carteggio del 1933, custodito dalla Fondazione luigi Einaudi di Torino, il fondatore della FIAT, Giovanni Agnelli, scrive al senatore Luigi Einaudi, futuro Presidente della Repubblica della necessità di ridurre l'orario di lavoro come rimedio a una disoccupazione causata da macchine sempre più produttive. Scrive Giovanni Agnelli: "Il progresso tecnico non avrebbe senso se dovesse servire a creare disoccupazione, crisi e malcontento sociale", Torino 10 gennaio 1933. Cf. (CAPOZZI, 2017, p. 34-37).

11 Cf. (BUCKLEY, 1967. p. 47). (trad. it., Sociologia e teoria dei sistemi. Torino: Boringhieri, 1976).

12 "L'apprendimento supervisionato è una tecnica di apprendimento automatico che mira a istruire un sistema informatico in modo da consentirgli di risolvere dei compiti in maniera autonoma sulla base di una serie di esempi ideali, costituiti da coppie di input e di output desiderati, che gli vengono inizialmente forniti." (WIKIPEDIA, 2017a, online).

13 "L'apprendimento non supervisionato è una tecnica di apprendimento automatico che consiste nel fornire al sistema informatico una serie di input (esperienza del sistema) che egli riclassificherà ed organizzerà sulla base di caratteristiche comuni per cercare di effettuare ragionamenti e previsioni sugli input successivi. Al contrario dell'apprendimento supervisionato, durante l'apprendimento vengono forniti all'apprendista solo esempi non annotati, in quanto le classi non sono note a priori ma devono essere apprese automaticamente." (WIKIPÉDIA, 2017b, online).

14 "L'apprendimento per rinforzo è una tecnica di apprendimento automatico che punta ad attuare sistemi in grado di apprendere ed adattarsi alle mutazioni dell'ambiente in cui sono immersi attraverso la distribuzione di una "ricompensa" detta rinforzo che consiste nella valutazione delle loro prestazioni. Può essere attuata mediante un algoritmo, come il Q-learning, da inserire all'interno del sistema in cui si vuole realizzare l'apprendimento." (WIKIPÉDIA, 2017c, online).

15 Nel campo dell'apprendimento automatico, una rete neurale artificiale (in inglese artificial neural network, abbreviato in ANN o anche come $\mathrm{NN}$ ) è un modello matematico composto di "neuroni" artificiali, che si ispira a una rete neurale. Questi modelli matematici possono essere utilizzati sia per ottenere una comprensione delle reti neurali biologiche, ma ancor di più per risolvere problemi ingegneristici di intelligenza artificiale come quelli che si pongono in diversi ambiti tecnologici (in elettronica, informatica, simulazione, e altre discipline). Una rete neurale artificiale può essere realizzata sia da programmi software che da hardware dedicato (DSP, Digital Signal Processing). Questa branca può essere utilizzata in congiunzione alla logica fuzzy. Cf. (KOSKO, 1992, 1993). Vedi anche: (WIKIPEDIA, 2017d).

16 L'Institute for the Future (IFTF) è un'organizzazione formata da un gruppo di esperti con sede a Palo Alto in California, fondata nel 1968 come spin-off dalla RAND Corporation, per aiutare le organizzazioni e le aziende a pianificare per il futuro a lungo termine (INSTITUTE FOR THE FUTURE, 2017a).

17 Per una interessante prospettiva della IA dal punto di vista dell'Informatica giuridica (SARTOR, [20--], p. 269 e ss).

18 Cf. ((INSTITUTE FOR THE FUTURE, 2017b).

19 L'innovazione tecnologica prodotta dalla rivoluzione informatica ha determinato la IV rivoluzione industriale coinvolgendo la produzione e l'industria che oggi viene definita 4.0 all'interno della quale si registra un uso notevole di robot. "Anche la linearità per stadi dello sviluppo industriale va problematizzata: la sequenza storica che parte dalla manifattura semplice, prosegue con il passaggio alla grande fabbrica e quindi al taylorismo, conduce al toyotismo e all'automazione flessibile per approdare infine alla smart factory è per molti versi un'astrazione, utile per rimarcare la discontinuità ma sottomessa all'idea che l'evoluzione dell'industria sia guidata secondo una direttrice unica. [...] Obiettivo esplicito delle smart factory è creare una catena linguistica in cui le informazioni vengano tradotte non più solo dai lavoratori ma anche dalle macchine". (MAGONE; MAZALI, 2016, p. 166-167).

20 Il World Economic Forum cita la robotica e l'intelligenza artificiale tra le venti tecnologie emergenti della Quarta Rivoluzione Industriale destinate a trasformare il mondo nei prossimi anni. Tale dato è confermato anche dalla Risoluzione del Parlamento europeo (Punto D) la quale evidenzia come "tra il $2010 \mathrm{e}$ il 2014 la crescita media delle vendite di robot era stabile al 17\% annuo e che nel 2014 è aumentata al 29\%, il più considerevole aumento annuo mai registrato, e che $i$ fornitori di parti motrici e l'industria elettrica/elettronica sono $i$ principali propulsori della crescita; che le richieste di brevetto per le tecnologie robotiche sono triplicate nel corso dell'ultimo decennio". Tuttavia il WEF evidenzia come tali tecnologie possano dare origine a grandi opportunità e, 
al contempo, a grandi rischi, il cui bilanciamento dipende dall'efficacia del sistema di regole, standard, incentivi, istituzioni e altri meccanismi volti a regolarne lo sviluppo e l'utilizzo. Dinanzi a questa esigenza di adeguamento del diritto, che non è ancora avvenuto, si possono osservare delle criticità. Vedi: (WORLD ECONOMIC FORUM, 2017).

21 Il 4 maggio 2016, sono stati pubblicati sulla Gazzetta Ufficiale dell'Unione Europea (GUUE) i testi del Regolamento europeo in materia di protezione dei dati personali e della Direttiva che regola $i$ trattamenti di dati personali nei settori di prevenzione, contrasto e repressione dei crimini. Il 24 maggio 2016 è entrato ufficialmente in vigore il Regolamento, che diventerà definitivamente applicabile in via diretta in tutti i Paesi UE a partire dal 25 maggio (EUROPA, 2016).

22 L'art. 14 del D.lgs. 196/2003, "Codice in materia di protezione dei dati personali", prevede il divieto della piena sostituzione di una macchina alla decisione umana che implichi profili giudiziari o amministrativi.

23 Il 5 aprile l'"Article 29 Data Protection Working Party" ha pubblicato le Linee Guida sulla Data Protection Impact Assessemnt (DPIA) al fine di dare una valida interpretazione all'art. 34 del Regolamento UE 2016/679. Le Linee Guida riflettono il punto di vista solo dell'Art. 29 WP che ha uno stato di consulenza e agisce in modo indipendente. Non riflettono la posizione della Commissione europea. <http://ec.europa.eu/newsroom/just/ item-detail.cfm?itemid=50083 > . Un DPIA è un processo progettato per descrivere l'elaborazione, valutare la sua necessità, la proporzionalità delle misure adottate e contribuire a gestire i rischi per i diritti e le libertà delle persone fisiche risultanti dall'elaborazione di dati personali, valutandoli e determinando le misure per affrontarli. Le DPIA sono importanti strumenti per imputare le responsabilità in quanto aiutano i controllori non solo a capire se sono soddisfatti i requisiti del GDPR, ma anche per dimostrare che sono state adottate misure adeguate per garantire la conformità con il Regolamento. In altre parole, una DPIA è un processo per la costruzione e la dimostrazione delle conformità <file://C:/Users/Utente/Desktop/20171013_wp248_rev01_enpdf.pdf>.

24 Dalla prospettiva dei sistemi esperti nel campo del diritto, sebbene datato, è utile la consultazione del volume: (MARIANI; TISCORNIA, 1989).

\section{REFERÊNCIAS}

BATTIFOGLIA, Enrica. I robot sono fra noi: dalla fantascienza alla realtà. Milano: Hoepli editore, 2016.

BUCKLEY, Walter. Sociology and modern systems theory. New York: Englewood Cliffs, 1967.

BURKEMAN, Oliver. The internet at 40: forty years of the internet: how the world changed for ever. The Guardian, United Kingdom, Oct. 2009. Available in: < https://www.theguardi an.com/technology/2009/oct/23/internet-40-history-arpanet $>$.

CAPOZZI, Fiorina. Carteggio Agnelli\&Einaudi. FQ Millennium, Mensile, anno 1, n. 7, p. 34-37, 2017.

EUROPA. Regolamento (UE) 2016/679 del Parlamento Europeo e del Consiglio. 2016. Disponibile in: <http://eur-lex.europa.eu/legal-content/IT/TXT/?uri=uriserv:OJ.L_.2016.119 01.0001.01.ITA\&toc $=$ OJ:L:2016:119:TOC>.

EUROPEAN PARLIAMENT. Civil Law Rules on Robotics: European Parliament resolution of 16 February 2017 with recommendations to the Commission on Civil Law Rules on Robotics (2015/2103(INL)). 2017a. Available in: <http:/www.europarl.europa.eu/ 
sides/getDoc.do?pubRef $=-/ / \mathrm{EP} / / \mathrm{TEXT}+\mathrm{TA}+\mathrm{P} 8-\mathrm{TA}-2017-0051+0+\mathrm{DOC}+\mathrm{XML}+\mathrm{V0} / /$ $\mathrm{EN}>$.

. Norme di diritto civile sulla robotica. Risoluzione del Parlamento europeo del 16 febbraio 2017 recante raccomandazioni alla Commissione concernenti norme di diritto civile sulla robotica (2015/2103(INL)). 2017b. Available in: <http://www.europarl.europa.eu/sides/ getDoc.do?pubRef=-//EP//TEXT + TA + P8-TA-2017-0051+0+DOC+XML+V0//IT > .

INSTITUTE FOR THE FUTURE. Home. 2017a. Disponibile in: <http://www.iftf.org/ home/>.

. Technologies Human-Machine. 2017b. Disponibile in: < http://www.iftf.org/fileadmin/userupload/downloads/th/SR1940IFTFforDellTechnologiesHuman-Machine070717readerhigh-res.pdf $>$.

ITALY. Unitelma Sapienza of Rome. National School of Digital Administration. IRIA Observatory (Internet of Things, Robotics and Artificial Intelligence). 2017. Available in: $<$ https://www.unitelmasapienza.it/it/snad $>$.

KOSKO, Bart. Fuzzy Thinking: the new science of fuzzy logic. New York: [s.n.], 1993.

. Neural Networks and Fuzzy Systems: a dynamical systems approach to machine intelligence. United States: Prentice-Hall International, 1992.

LUHMANN, Niklas. La differenziazione del diritto. Bologna: il Mulino, 1990.

MAGONE, Annalisa; MAZALI, Tatiana. Industria 4.0: uomini e macchine nella fabbrica digitale. Milano: Guerini e associati, 2016.

MARIANI, Paola; TISCORNIA, Daniela. Sistemi esperti giuridici: l'intelligenza artificiale applicata al diritto. Milano: Franco Angeli, 1989.

MARINI, Luca; APREA, Isabella. Le guidelines on regulating robotics: una sfida per il diritto dell'Unione. Ordine Internazionale e Diritti Umani, Roma, n. 5, p. 1295-1302, 2015. MARRO, Enrico. Allarme Onu: i robot sostituiranno il 66\% del lavoro umano. 2016. disponibile in: <http://nova.ilsole24ore.com/nova24-tech/allarme-onu-i-robot-sostituiranno-il-66-del-lavoro-umano/>.

MORO, Paolo. Libertà del robot? Sull'etica delle macchine intelligenti. In: BRIGHI, Raffaella; ZULLO, Silvia. (a cura di). Filosofia del diritto e nuove tecnologie: prospettive di ricerca tra teoria e pratica. Ariccia: Aracne, 2015. p. 525-544.

MOSCA, Giuditta. L'Arabia Saudita dà la cittadinanza all'androide Sophia. 2017. Disponibile in: <https:/www.wired.it/attualita/tech/2017/10/30/arabia-saudita-cittadinanza-sophia/>. ROBOTS and Industrialization in Developing Countries. Policy Brief, n. 50, Oct. 2016. Available in: <http://unctad.org/en/PublicationsLibrary/presspb2016d6en.pdf $>$. 
ROMANDINI, Marco, Condannato da un tostapane. FQ Millennium, Mensile, anno 1, n. 7, p. 21-31, 4 nov. 2017.

SANTOSUOSSO, Amedeo; BOSCARATO, Chiara; CAROLEO, Floro. Robot e diritto: una prima ricognizione. La Nuova Giurisprudenza Commentata, n. 7-8, p. 494-516, 2012. SARTOR, Giovanni. L'informatica giuridica e le tecnologie dell'informazione. Torino: Giappichelli, [20--].

SEARLE, John R. Minds, Brains and Programs. The Behavioral and Brains Sciences, n. 3, p. 417-457, 1980.

STANZIONE, Pasquale. Biodiritto, postumano e diritti fondamentali. Comparazione e Diritto Civile, Salerno, p. 1-15, maggio 2010. Disponibile in: < https://www.compara zionedirittocivile.it/prova/files/stanzionebiodiritto.pdf $>$.

WIKIPÉDIA. Apprendimento Supervisionato. 2017a. Disponibile in: < https://it.wikipedia. org/wiki/Apprendimento_supervisionato $>$.

. Apprendimento non supervisinato. 2017b. Disponibile in: < https://it.wikipedia. org/wiki/Apprendimento_non_supervisionato $>$.

. Apprendimento per rinforzo. 2017c. Disponibile in: < https://it.wikipedia.org/ wiki/Apprendimento_per_rinforzo $>$.

. Reteneurale artificiale. 2017d. Disponibile in: < https://it.wikipedia.org/wiki/Reteneurale_artificiale $>$.

WORLD ECONOMIC FORUM. The Future of jobs. 2016. Available in: < http://www3. weforum.org/docs/WEF_Future_of_Jobs.pdf $>$.

. The Global Risks Report 2017. 2017. Available in: <https://www.weforum.org/ reports/the-global-risks-report-2017>.

Data de submissão: 3 jan. 2018

Data de aprovação: 20 fev. 2018 